\title{
Performance Evaluation of MANET Routing Protocols for Varying Topologies Size
}

\author{
Awos Kh. Ali \\ Department of Computer Science / College of Education \\ University of Mosul
}

Received
$29 / 09 / 2010$
Accepted

02 / 03 / 2011

\section{الخلاصة}

شبكة المحمول (ad-hoc) هي مجموعة من الأجهزة النقالة المتصلة فيما بينها بشبكة

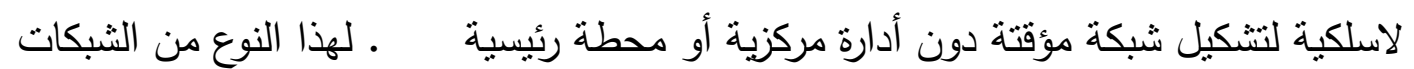
اللاسلكية سمات متل اتصال لاسلكي وتغيير مستمر في هيئة الثبكة وسهولة أنتشار الأجهزة

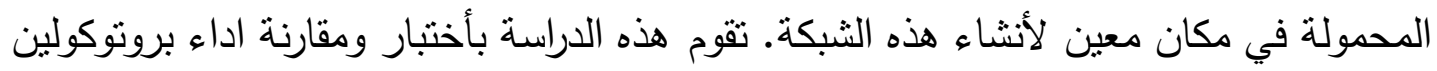

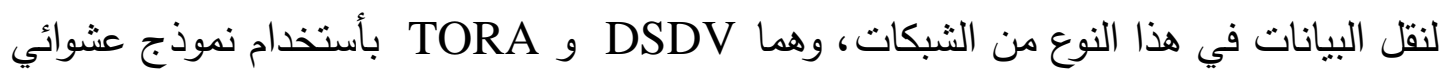
لحركة الأجهزة الدحمولة، في هذه الدراسة تم اختيار اثثين من مقاييس الأداء ، وهما معدل تأخير وصول البيانات و نسبة البيانات الواصلة للجه از المستقبل ـ تم أختبار البروتوكولين ببرنامج

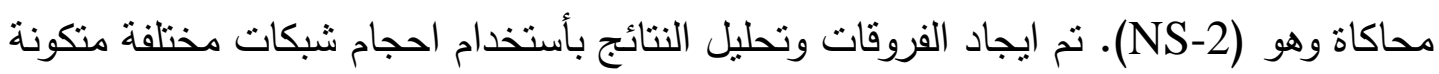

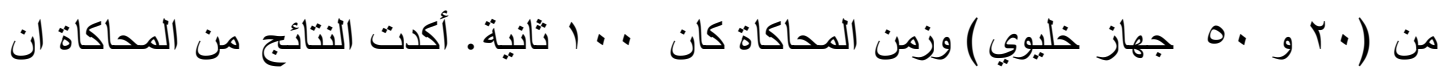

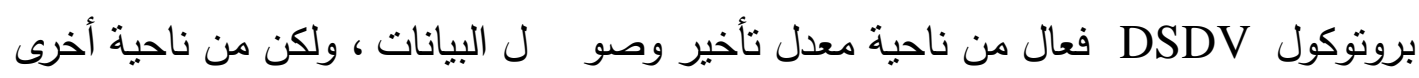
بروتوكول TORA كان أفضل من DSDV من ناحية نسبة البيانات الواصلة للجهاز المستقبل والتغير المستمر بهيئة الثبكة.

\section{ABSTRACT}

Mobile ad-hoc network (MANET) is a collection of wireless mobile hosts forming a temporary network without the centralized administration or base station. Mobile ad-hoc network have the attributes such as wireless connection, continuously changing topology, ease of deployment. This study has compared the performance of two MANET routing protocol DSDV and TORA by using random mobility model. In this study two performance metrics have been chosen, such as Average 
Delay and throughput. The simulations are carried out on NS-2. The performance differentials are analyzed using varying network size (20 and 50 nodes) and simulation time was 100s. Simulation results confirm that DSDV performs well in terms of Average Delay. But TORA performs better than DSDV in terms of throughput and changing in network topology.

\section{Introduction}

Mobile ad hoc networks (MANETs) consist of a set of mobile devices typically referred to as nodes (hosts) that are connected by wireless link without pre-existing network infrastructure or centralized base station [1]. These nodes can be arbitrarily located and are free to move randomly at any given time and any where, thus allowing network topology and interconnections between nodes to change rapidly. Node mobility can vary between network to other depending on the particular network's structure and purpose. Wireless networks have become popular in the past few years, when they are being adapted to enable mobility and wireless devices such as Mobile hand phone and laptop computers. In an ad hoc network, all nodes are required to relay packets for other nodes in order to deliver data across the network. There is no router like in wired network, thus the node itself operate the router job [2]. As a general rule, high mobility usually results in low link capacity between nodes, whereas low mobility leads to high capacity links. An ad hoc network is usually a self-organizing and self-configuring "multi-hop" network which does not require any fixed infrastructure such as transceiver base stations or even cables [3].

Ad hoc networks are suited for use in situations where infrastructure is either not available, not trusted, or should not be relied on in times of emergency. A few examples include: military solders and equipments in the battlefield ("open environment"), sensor networks for various research purposes, emergency rescue after an earthquake or flood, and temporary offices, conference or meeting in such company ("close or limited environment") [4].

\section{Routing in MANET:}

Routing can be defined as the process of information exchange from one node to the other node in a network.'[3]. Routing is the mechanism of forwarding packet towards its destination using most optimum path. Efficiency of the path can be measured in various metrics like; Number of hops, traffic, and delay of delivered the packets from source node to destination, etc [5].

Routing can be classified into static routing and dynamic routing. Static routing refers to the routing strategy being stated manually or 
statically, in the router. Static routing maintains a routing table usually written by a networks administrator. The routing table does not depend on the state of the network status, i.e., whether the destination is active or not [4]. Dynamic routing refers to the routing strategy that is being learnt by routing protocol. This routing depends on the state of the network i.e., the routing table is affected by the activeness of the destination [3].

The large variety of routing protocols reflects the fact that these protocols do implement strategies very differently [6]. The following section focuses on some types of ad hoc routing protocols, and their classifications.

\section{Categorization of routing protocols in MANET:}

Routing protocols for mobile ad-hoc networks can be classified into three major categorizes, based on the routing information update mechanism. They are:

1. Table Driven routing Protocols (Proactive).

2. On Demand routing Protocols (Reactive).

3. Hybrid routing protocols.

Figure 1 illustrates the classification of ad-hoc routing protocols [1].

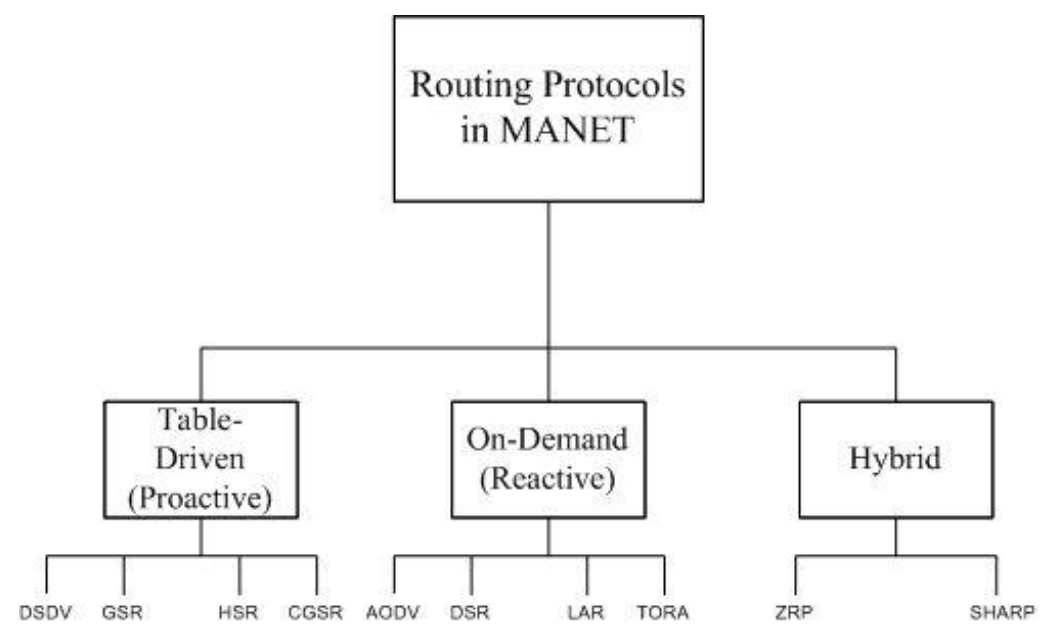

Figure 1: Classification of ad-hoc routing protocols.

\subsection{Table Driven Routing Protocols (Proactive):}

Proactive routing protocols are extensions of the wired network routing protocols. They maintain the global topology information into the tables at every node in the network. These tables are updated frequently in order to maintain the network topology changes. The update routing table process done by exchanging the recent update information from the other nodes in the same network. The exchanging information is happened by broadcasting the messages between nodes. These protocols are also called as proactive protocols since they maintain the routing 
information even before it is needed [7]. The proactive protocols are not suitable for larger networks, because this causes more overhead in the routing table leading to consumption of more bandwidth and memory [2]. Examples of Proactive Routing Protocols are:

- Destination Sequenced Distance Vector Routing (DSDV).

- Global State Routing (GSR).

- Hierarchical State Routing (HSR).

- Cluster head Gateway Switch Routing (CGSR).

The following section describe destination sequenced distance vector routing protocol (DSDV) which is one of routing protocol that route data between the nodes within wireless network using proactive strategy.

Destination sequenced distance vector routing protocol (DSDV) is one of the first protocols proposed for ad-hoc wireless networks. It is an enhanced version of the distributed Bellman-Ford algorithm. Where each node maintains a table that contains the shortest path to the all nodes within the network, and the number of hops to these nodes. So each entry to this routing table labeled with sequence number by destination node. The mobile node can recognize the old route from new one through this sequence number. This property can help to avoiding the routing loop problem. The routing table is periodically updated through the neighbor's nodes. The packet used by nodes to update the routing table can be classified into two possible types of packets, the first kind called a "full dump", this type of packets contain all available routing information about others nodes, the second type is smaller than first one, is called "incremental". This kind of packets carried only the information about topology changes since last full dump, and this packet decreasing the traffic on network. However, the information about new route could be broadcasted or multicast [8]. The packet of this information contain the address of the destination, the number of hops to reach the destination, the sequence number of the information received regarding the destination, as well as a new sequence number unique to the broadcast [5].

\subsection{On Demand Routing Protocols (Reactive):}

Unlike the table-driven (proactive) routing protocols, on-demand routing protocols execute the path finding process and exchange routing information only when a path is required. When a node requires a route to destination node, it is initiates a route discovery process within the network, when a route found or many possible routes discovered then the node choose a specific route with smaller metric (shortest path). In this approach of routing (Reactive) try to eliminate the routing tables and reduce the need of updating these tables in contrast with proactive (table 
driven) routing protocols which maintain all tables up-to-date at every node. in reactive routing protocols (on demand) routes is always available with reduce of network traffic and power consumption but on demand routing suffer longer delay while route discovery [9].

Examples of reactive protocols are:

- Ad hoc On-demand Distance Vector Routing (AODV).

- Dynamic Source Routing (DSR).

- Location Aided Routing (LAR).

- Temporally Ordered Routing Algorithm (TORA) [7].

The following section describe temporally ordered routing protocol (TORA) which is one of the routing protocol that route data between the nodes within wireless network using reactive strategy.

Temporally ordered routing algorithm (TORA) is a source initiated on-demand routing protocol with uses a link reversal algorithm and provides a loop free multi path routes to destination node.

TORA is suitable for high dynamic mobile networking environment; the concept of TORA is localization of control message to a very small group of nodes, this protocol perform three basic function, route creation, route maintaining, and route erasing [8].

In TORA, the network topology is considered as a directed graph. During the route creation and maintenance phases, nodes use a height metric to create a Directed Acyclic Graph (DAG) rooted at the destination, after that links are assigned a direction upstream or downstream, depend on the relative height metric of neighboring nodes, This process of establishing a DAG is similar to the query reply process. Despite, some times of node mobility the DAG route is broken and route maintenance is necessary to re-establish a DAG rooted at the same destination [2].

Node generates a new reference level which results in the propagation of that reference level by elective neighboring nodes coordinating a structured reaction to the failure Links are reversed to the change in adapting to the new reference level; this has the same effect as reversing the direction of one or more links when a node has no downstream links.

When multiple nodes wants erasing routes and building new routes based on each other, its instability problem is similar to the count to infinity problem in distance vector routing protocols and routes cross will occur [9].

\subsection{Hybrid Routing Protocols:}

The ad hoc network can use the hybrid routing protocols that have the advantage of both proactive and reactive routing protocols to balance the 
delay and control overhead (in terms of control packages). Hybrid routing protocols try to maximize the benefit of proactive routing and reactive routing by utilizing proactive routing in small networks (in order to reduce delay), and reactive routing in large-scale networks (in order to reduce control overhead) [2].

The difficulty of all hybrid routing protocols is how to organize the network according to network parameters. The common disadvantage of hybrid routing protocols is that the nodes that have high level topological information maintains more routing information, which leads to more memory and power consumption [10].

\section{Simulation Model:}

The simulation experiment is carried out in LINUX (REDHAT 5). The detailed simulation model is based on network simulator-2 (ver2.30), is used in the evaluation. The following section describe NS2 (network simulator) in detail.

NS2 is an object-oriented, discrete event packet level network simulator. It is usually used by the academic community to evaluate research proposals or ideas in the area of networking. Furthermore, NS2 use both OTcl script and $\mathrm{C}++$ to implement a protocols and setup and direct simulations scenarios. $\mathrm{C}++$ is often used when control is needed at packet level. In the same time OTcl is a scripting language, that allows for the connecting of various other languages (originally $\mathrm{C}$, now also C++, Java, Eiffel, and Prolog). Scripts can be written to allow the bringing together of a number of different sections of code in these languages [11].

Simulation has been done by using two kinds of scenario for both protocols, DSDV and TORA; the first scenario done using 20 nodes and the second scenario with 50 nodes. The simulation period for each scenario is 100 seconds, and the simulated mobility network area is 1000 $\mathrm{m} \times 1000 \mathrm{~m}$ rectangle. In each simulation scenario, the nodes are move randomly. The following table illustrates the simulation setup.

Table 1: Simulation setup

\begin{tabular}{|l|l|}
\hline \multicolumn{1}{|c|}{ Simulation parameter } & \multicolumn{1}{c|}{ Value } \\
\hline Channel type & Wireless Channel \\
\hline MAC type & 802.11 \\
\hline Link layer type & Traditional Link Layer (LL) \\
\hline Antenna model & Omni-directional (unity gain) \\
\hline No. of nodes & 20 and 50 \\
\hline No. of sending nodes & 1 \\
\hline Mobility & Random way point \\
\hline Routing Protocol & DSDV and TORA \\
\hline No. of receive nodes & 1 \\
\hline Simulation time & $100 \mathrm{Sec}$ \\
\hline Size of simulation area & $1000 \mathrm{~m} \mathrm{x} 1000 \mathrm{~m}$ \\
\hline Initial Power level & $104000 \mathrm{watt}$ \\
\hline
\end{tabular}


Figure 2: and figure 3 illustrate the network topology that used in the evaluation for both scenarios ( 20 and 50 nodes).

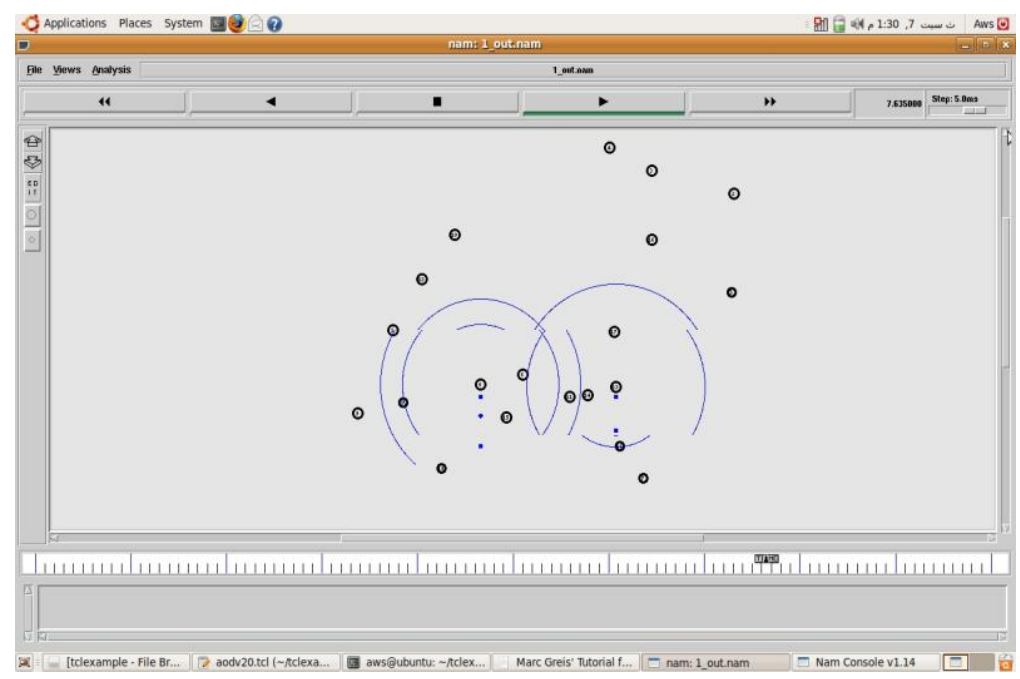

Figure 2: 20 nodes Network Topology.

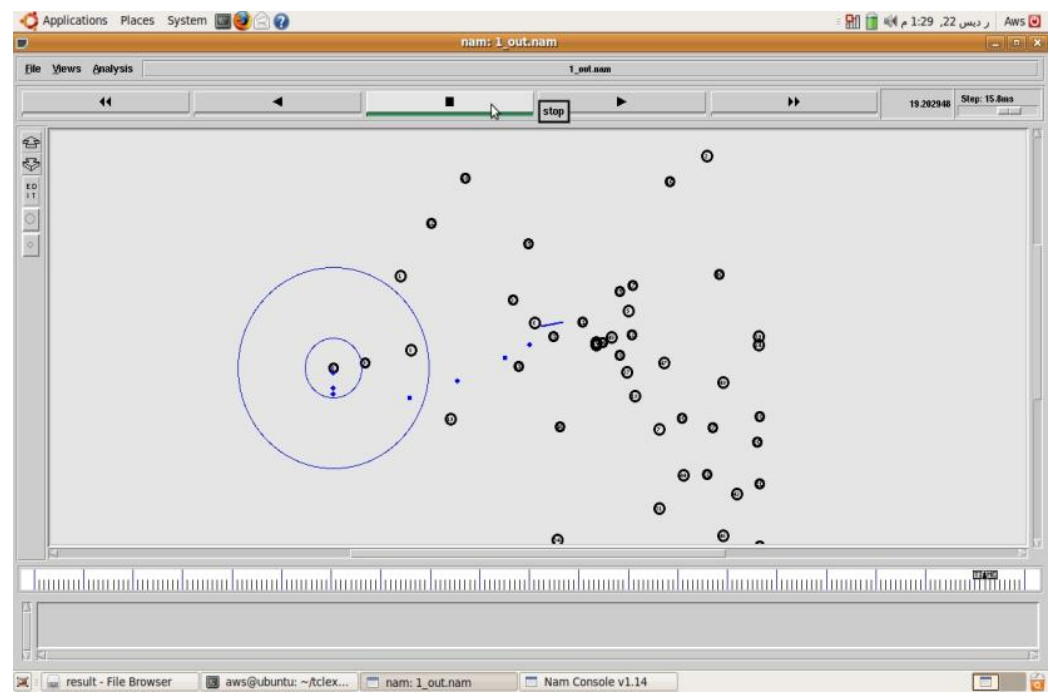

Figure 3: 50 nodes Network Topology.

\subsection{Performance Metric}

There are different kinds of metric for the performance evaluation of the routing protocols. These have different behaviors of the overall network performance. This study will evaluate two metric for the comparison of the overall network performance. These metric are delay, and throughput for protocols evaluation. These metric are important in the consideration of evaluation of the routing protocols in a communication network. These protocols need to be checked against certain parameters for their performance. To check protocol effectiveness in finding a route towards destination, if the routing protocol gives much end to end delay so probably this routing protocol is not efficient in big 
network topology as compare to the protocol which gives low end to end delay. Similarly the same is the case with the throughput as it represents the successful deliveries of packets in time. If a protocol shows high throughput so it is the efficient and best protocol than the routing protocol which have low throughput. These parameters have great influence in the selection of an efficient routing protocol in any communication network.

\subsection{Delay}

The packet end-to-end delay is the time of generation of a packet by the source node until reach the destination node. So this is the time that a packet takes to go across the network. This time is expressed in sec. Hence all the delays in the network are called packet end-to-end delay, like buffer queues and transmission time. Sometimes this delay can be called as latency; it has the same meaning as delay. Some applications are sensitive to packet delay such as voice is a delay sensitive application. So the voice requires a low average delay in the network [8].

\subsection{Throughput}

Throughput is defined as; the ratio of the total data reaches a receiver from the sender. The time it takes by the receiver to receive the last message is called as throughput [11][12]. Throughput is expressed as bytes or bits per sec (byte/sec or bit/sec). Some factors affect the throughput as; if there are many topology changes in the network, unreliable communication between nodes, limited bandwidth available and limited energy [13]. A high throughput is absolute choice in every network. Throughput can be represented mathematically as in the following equation [1][14][15].

Throughput $=\frac{\text { Number of delivered packets } * \text { packet size } * 8}{\text { total duration of simulation }}$

\section{Results and Discussion}

In this section the simulation results are presented and discussed in detail .This results is used in order to evaluate the network performance of the two routing protocols DSDV and TORA. First, an attempt was made to compare the two protocols under the same simulation environment. For all the simulations, the same movement models were used (random way point), the number of nodes was fixed at 20 and 50 for each scenario, the maximum speed of the nodes was set to $25 \mathrm{~m} / \mathrm{s}$ and the simulation time was 100 s.

The following graphs (4 and 5) illustrate the throughput of DSDV and TORA protocols with 20 nodes scenario; 


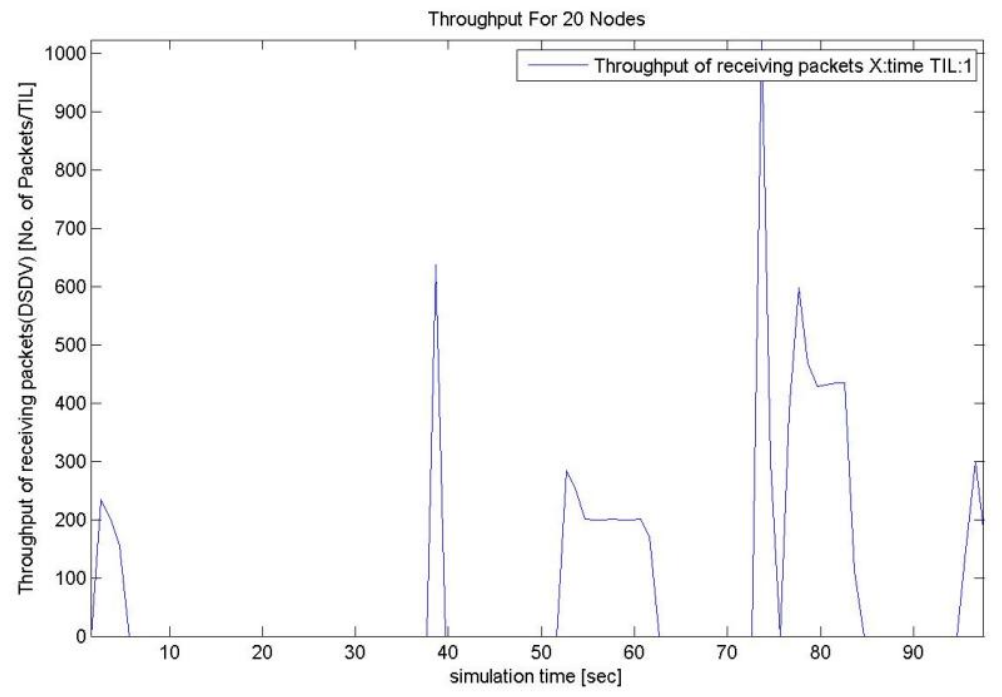

Graph 4: Throughput of DSDV (20 nodes)

In 20 nodes Scenario, the routing protocol DSDV was compare based on its throughput. Graph 4 shows throughput of DSDV routing protocol, which is too weak especially when the topology of networks rapidly changing. This limitation because of DSDV does not support multi path routing to the destination node, and difficulty to maintain the routing table's advertisement for high dynamic topology.

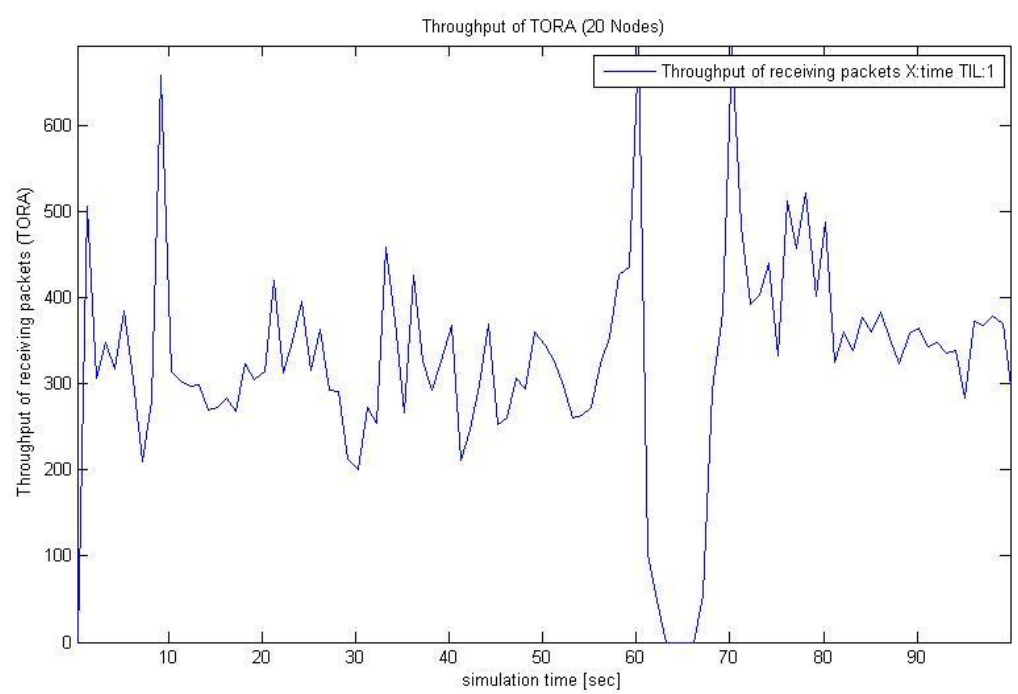

Graph 5: Throughput of TORA (20 nodes)

Graph 5 shows the throughput of TORA routing protocol in same kind of scenario (20 nodes Scenario). TORA perform well in high dynamic topology, which is better than DSDV; because TORA Support 
multiple routes to destination node. So, in case of that the route between source and destination is disconnect, the source node use another route.

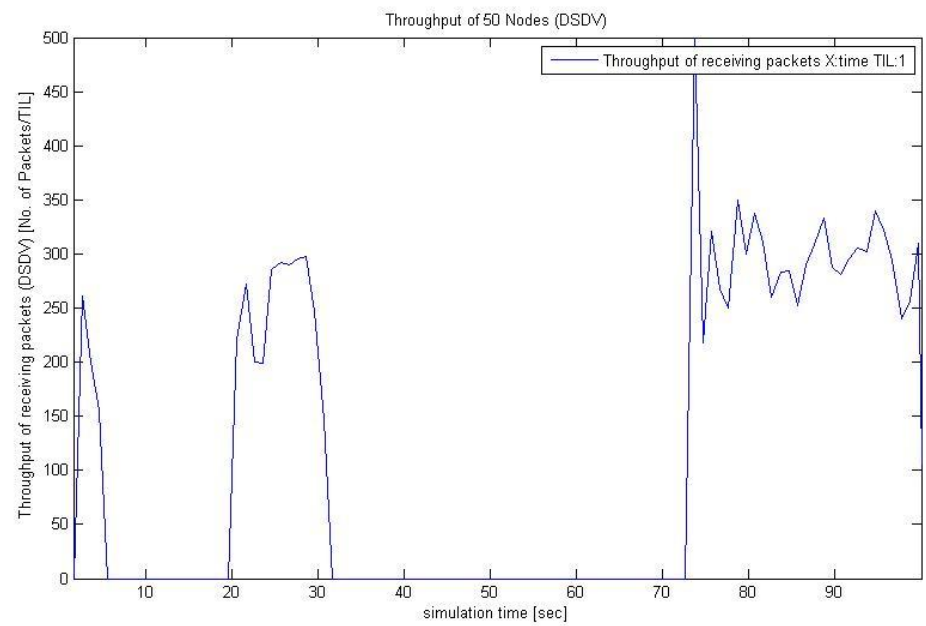

Graph 6: Throughput of DSDV (50 nodes)

In 50 nodes scenario, graphs 6 illustrate the throughput of DSDV, that still weak because of the same reason that mention before (DSDV does not support multi path routing to the destination node, and difficulty to maintain the routing table's advertisement for high dynamic topology).

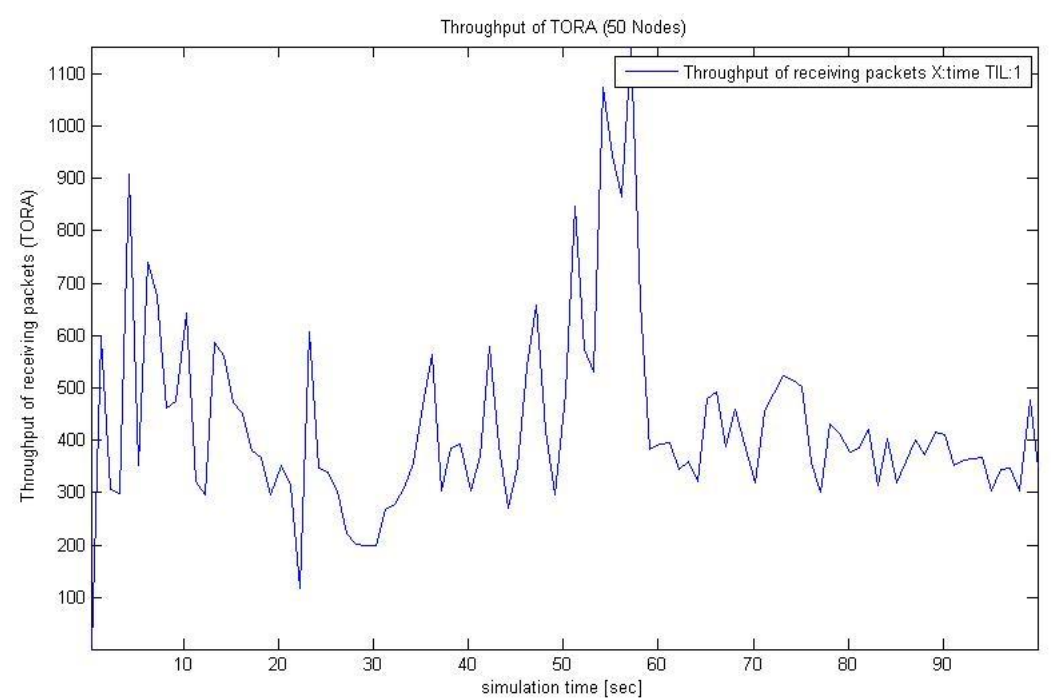

Graph 7: Throughput of TORA (50 nodes)

Graph 7 illustrate the throughput of TORA routing protocol with same kind of scenario (50 nodes scenario), it can be observed that throughput of TORA is better than DSDV as well. Due to enhance the routing mechanism of choosing multi path to destination node and deal with high dynamic topology environment. 
On the other hand, in high mobility scenario, link failure can be happened very frequently. Link failures start new route discoveries process in TORA. So on varying speed of nodes the packet end to end delay will increases because the increasing of the node speed, the link between source and destination will break frequently, (Graph 8) illustrate end to end delay with 20 nodes scenario. DSDV has a better Average Delay than TORA, The reason for this is that it finds routes faster or that the routes are shorter or more optimal; because DSDV has all routes to all nodes in the network in routing table, in reverse with TORA.

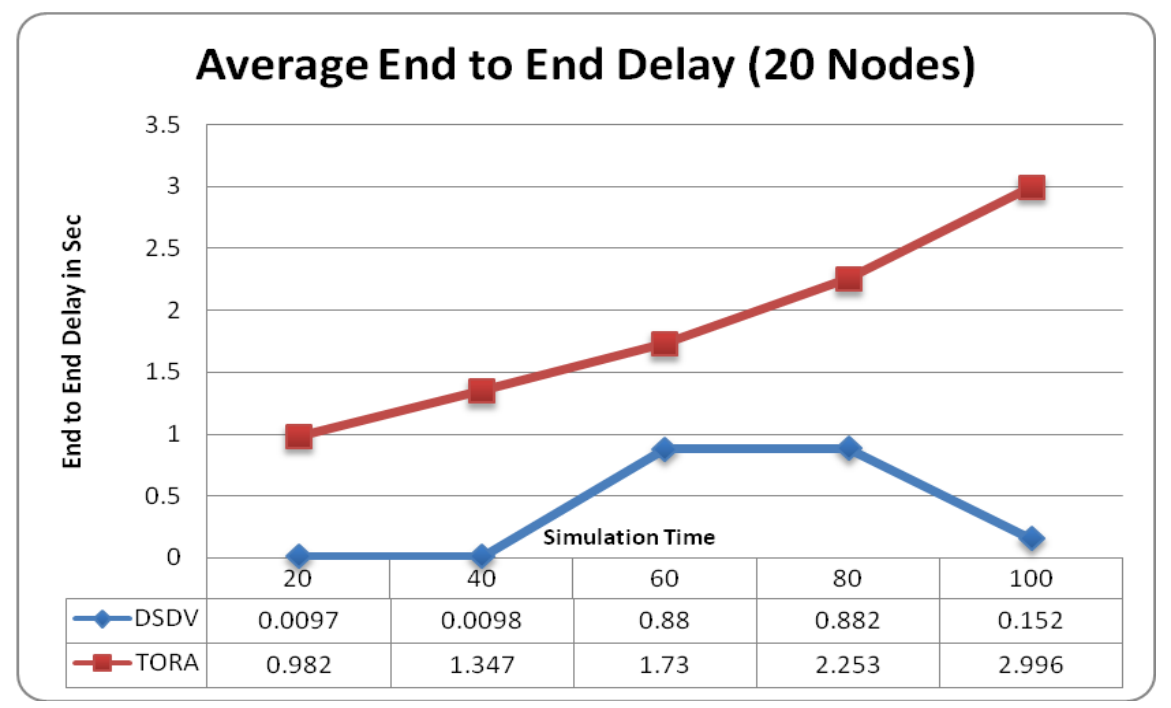

Graph 8: Average End to End Delay(20 nodes)

In stressful situation (50 nodes scenario), average delay of DSDV protocol still smaller than TORA protocol. TORA perform well in all conditions especially in high dynamic topology; but DSDV shows good average delay compare with TORA. Graph 9 illustrates average end to end delay with 50 nodes scenario.

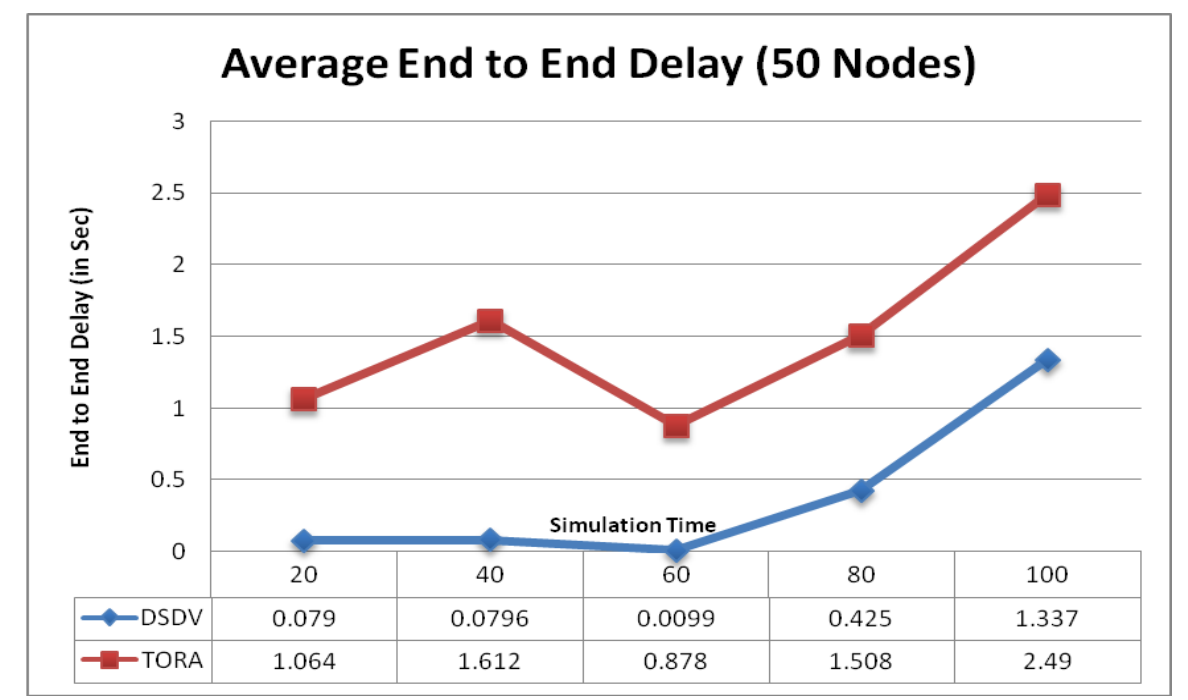

Graph 9: Average End to End Delay(50 nodes) 


\section{Conclusion:}

In this study, two routing protocol (DSDV and TORA) are tested and their performance have been analyzed under random mobility model with respect to two performance metrics (Throughput and Average end to end delay). The simulation results shows average end to end delay of DSDV less than TORA in both type of scenario (20 and 50 nodes), but the performance of TORA protocol in term of throughput is better than DSDV in both type of scenario ( 20 and 50 nodes) as well.

\section{REFRENCES}

1) Ali A, Ali S, 2009, M. Sc. Thesis "Performance Analysis of AODV, DSR and OLSR in MANET Department "Telecommunication Blekinge Institute of Technology, Sweden.

2) Abdullah A, Ramly N, Muhammed A, Derahman MN, 2008, "A PERFORMANCE STUDY OF ROUTING PROTOCOLS FOR MOBILE GRID ENVIRONMENT", Journal of ICT, 8, pp: 41-54.

3) Bindra H S, Maakar S K, Sangal A L, 2010, "Performance Evaluation of Two Reactive Routing Protocols of MANET using Group Mobility Model", Department of Computer Science and Engineering National Institute of Technology, Jalandhar (Punjab) 144011, India.

4) Azad Md. S, Uddin M M, Anwar F, Rahman Md. A, 2008, "Performance Evaluation of Wireless Routing Protocols in Mobile WiMAX Environment", Proceedings of the International MultiConference of Engineers and Computer Scientists, Hong Kong.

5) Ducatelle F, Di Caro G, Gambardella LM, 2008, "A New Approach for Integrating Proactive and Reactive Routing in Mobile Ad Hoc Networks".

6) El-Sayed H.M., Jaseemuddin M, Qureshi U, Bazan O, 2010, "PERFORMANCE EVALUATION OF TCP IN MOBILE ADHOC NETWORKS", WINCORE Lab, Ryerson University, Toronto, Ont., Canada.

7) Giannoulis S, Antonopoulos C, Topalis E, Koubias S, 2010, "ZRP versus DSR and TORA: A comprehensive survey on ZRP performance", Dept of Electrical and Computer Engineering, University of Patras Rion Campus, Patras, Greece. 
8) Lopez P, Prodanoff Z, Ahuja S, 2008, "Performance Evaluation of Routing Protocols for Mobile Ad-Hoc Networks (MANETs)", University of North Florida, USA.

9) Khatri P, Rajput M, Shastri A, Solanki K, 2010, "Performance Study of Ad-Hoc Reactive Routing Protocols", Journal of Computer Science 6 (10): 1130-1134, India.

10) Klein A, 2010, "Performance Comparison and Evaluation of AODV,OLSR, and SBR in Mobile Ad-Hoc Networks", EADS Deutschland GmbH Munich, Germany.

11) Suman A, Nagar A K, Jain S, Saurav P, 2010, "Simulation Analysis of STAR, DSR and ZRP in Presence of Misbehaving Nodes in MANET", Bhopal, India.

12) Uyen T N, Xing X, 2007, "Rate-adaptive Multicast in Mobile Adhoc Networks, Department of Computer Science and Engineering York University Toronto, Canada M3J 1P3.

13) Vetrivelan N, Reddy A V, 2008, "Performance Analysis of Three Routing Protocols for Varying MANET Size" Proceedings of the International MultiConference of Engineers and Computer Scientists, Vol II.

14) Duniel L. (2003). A comprehensive overview about selected Ad Hoc Networking Routing Protocols.

15) Gowrishankar S, Basavaraju T.G., Singh M, Sarkar S K, 2010, "Scenario based Performance Analysis of AODV and OLSR in Mobile Ad hoc Networks", Jadavpur University, Acharya Institute of Technology India. 